\title{
Veröffentlicht als:
}

Wessler, Hartmut und Eike Mark Rinke. 2013. "Öffentlichkeit.” In Handwörterbuch zur Gesellschaft Deutschlands, herausgegeben von Steffen Mau und Nadine M. Schöneck, 3. Aufl., 637-50. Wiesbaden: Springer VS.

Dies ist ein angenommenes Manuskript („Postprint“) eines Buchkapitels, das von Springer VS 2013 im „Handwörterbuch zur Gesellschaft Deutschlands“ veröffentlicht wurde. Die finale Version ist hier verfügbar: https://doi.org/10.1007/978-3-531-18929-1_43 


\section{Öffentlichkeit}

Hartmut Wessler, Eike M. Rinke

1. Begriffsbestimmung und Theorietraditionen

Öffentlichkeit ist ein sozialer Raum, in dem unterschiedliche Erfahrungen und Meinungen ausgedrückt, allgemein interessierende Fragen diskutiert und kollektive Lösungen kommunikativ entwickelt werden. In Flächenländern wie der Bundesrepublik Deutschland sind die Massenmedien (Zeitungen, Zeitschriften, Fernsehen, Radio) und seit Ende der 1990er Jahre auch Online-Medien (Webseiten, soziale Netzwerke, Diskussionsforen, Blogs etc.) die zentralen Foren öffentlicher Kommunikation. Aufgrund der Vielzahl der Foren wird Öffentlichkeit zumeist als ein Netzwerk aus Teilöffentlichkeiten aufgefasst, die sich jedoch vielfach gegenseitig beobachten. Medial vermittelte Öffentlichkeiten werden auch heute noch ergänzt durch Versammlungsöffentlichkeiten (Podiumsdiskussionen, Protestveranstaltungen etc.) und informelle Encounters in öffentlichen oder halböffentlichen Räumen (Gespräche in der Warteschlange, am Arbeitsplatz etc.) (Gerhards 1993). Öffentlichkeit wird hergestellt durch die Äußerungen unterschiedlicher Sprechertypen (Neidhardt 1994; Peters 2007: 76f.): Repräsentanten sind Sprecher, die individuelle oder organisationale Eigeninteressen vertreten, seien sie nun im Zentrum des politischen Systems angesiedelt (Akteure aus Regierung, Parlament, politischen Parteien) oder in dessen Peripherie (Verbände, Gewerkschaften, Unternehmen, neue soziale Bewegungen, einfache Bürger auf der Inputseite, Judikative und Verwaltungen auf der Outputseite des politischen Systems). Neben solchen Repräsentanten melden sich in der Öffentlichkeit Advokaten (Fürsprecher für die Interessen unterprivilegierter oder nichtartikulationsfähiger Gruppen), Experten und Intellektuelle zu Wort. Auch Journalisten sind Öffentlichkeitssprecher eigenen Rechts, wenn sie öffentliche Debatten nicht nur beschreiben, sondern durch Kommentare selbst eingreifen.

Der Begriff der Öffentlichkeit vereinigt empirische und normative Bedeutungsgehalte. In empirischer Hinsicht geht es in der Öffentlichkeitsanalyse um die Untersuchung der tatsächlich vorfindbaren Strukturen, Akteure und Prozesse der allgemein sichtbaren Meinungsbildung. Die empirische Öffentlichkeitsanalyse umfasst daher vieles von dem, was in der Kommunikations- und Medienwissenschaft und dort vor allem im Hinblick auf politische Kommunikation untersucht wird. In normativer Hinsicht verbinden sich mit dem Begriff der Öffentlichkeit bestimmte Sollensvorstellungen über das Funktionieren öffentlicher 
Meinungsbildung, die sich aus unterschiedlichen normativen Vorstellungen von Demokratie speisen. Normative Öffentlichkeitsvorstellungen geben ideale Merkmale öffentlicher Kommunikation an, spezifizieren Bedingungen ihrer Verwirklichung und ermöglichen eine kritische Bewertung tatsächlicher Kommunikationsverhältnisse. In Deutschland erhielt die normative Auseinandersetzung mit Öffentlichkeit einen entscheidenden Impuls durch das 1962 erschienene Buch „Strukturwandel der Öffentlichkeit“ von Jürgen Habermas (Habermas 1990), das nach seiner späten Übersetzung ins Englische 1989 auch international viel Resonanz ausgelöst hat (Calhoun 1992). In dieser Studie interpretiert Habermas die Entwicklung von Öffentlichkeit seit dem 18. Jahrhundert als Degenerationsprozess von einer kritisch-räsonierenden zu einer auf pure Akklamation durch das Massenpublikum abzielenden vermachteten Öffentlichkeit. Diese kulturkritische Verfallsthese hat Habermas selbst im Vorwort zur Neuauflage 1990 relativiert und in mehreren Schritten zu einem Modell deliberativer Öffentlichkeit weiterentwickelt (Habermas 1992, 2008; vgl. auch Peters 2007). Heute lässt sich das breite Feld unterschiedlicher normativer Öffentlichkeitstheorien vereinfachend in drei wesentliche Traditionen unterteilen: die liberale, die deliberative und die agonistische Tradition (Tabelle 1).

Tabelle 1: Normative Anforderungen an Öffentlichkeit: Drei Traditionen

\begin{tabular}{|c|c|c|c|c|}
\hline & $\begin{array}{l}\text { Zentrale } \\
\text { Metapher(n) } \\
\text { für } \\
\text { Öffentlichkeit }\end{array}$ & $\begin{array}{l}\text { Wer soll } \\
\text { kommunizieren? }\end{array}$ & $\begin{array}{l}\text { Wie soll } \\
\text { kommuniziert } \\
\text { werden? }\end{array}$ & $\begin{array}{l}\text { Mit welchem } \\
\text { Ergebnis soll } \\
\text { kommuniziert } \\
\text { werden? }\end{array}$ \\
\hline $\begin{array}{l}\text { Liberale } \\
\text { Tradition }\end{array}$ & Spiegel & $\begin{array}{l}\text { Repräsenta- } \\
\text { tionsmodell: } \\
\text { Vertreter gesell- } \\
\text { schaftlicher } \\
\text { Gruppen } \\
\text { (proportional) }\end{array}$ & $\begin{array}{l}\text { Verschiedene } \\
\text { Kommunikations- } \\
\text { stile zugelassen } \\
\text { (unter Wahrung } \\
\text { hinreichenden } \\
\text { Respekts) }\end{array}$ & $\begin{array}{l}\text { Abschluss der } \\
\text { Debatte nach } \\
\text { Feststellung des } \\
\text { Mehrheitsinteresses }\end{array}$ \\
\hline $\begin{array}{l}\text { Deliberative } \\
\text { Tradition }\end{array}$ & $\begin{array}{l}\text { Diskussions- } \\
\text { runde }\end{array}$ & $\begin{array}{l}\text { Partizipations- } \\
\text { modell: } \\
\text { Einschluss aller } \\
\text { betroffenen } \\
\text { Gesellschafts- } \\
\text { gruppen }\end{array}$ & $\begin{array}{l}\text { Dialog, Respekt, } \\
\text { Begründung, } \\
\text { Zivilität }\end{array}$ & $\begin{array}{l}\text { Abschluss durch } \\
\text { argumentativ } \\
\text { gestützte } \\
\text { Mehrheitsmeinung } \\
\text { (oder Konsens) }\end{array}$ \\
\hline $\begin{array}{l}\text { Agonistische } \\
\text { Tradition }\end{array}$ & Protestmarsch & $\begin{array}{l}\text { Empowerment- } \\
\text { modell: } \\
\text { Einschluss aller, } \\
\text { vor allem auch } \\
\text { subalterner } \\
\text { Gruppen }\end{array}$ & $\begin{array}{l}\text { Narration, } \\
\text { Selbstexpression, } \\
\text { Rhetorik }\end{array}$ & $\begin{array}{l}\text { Vermeidung des } \\
\text { Abschlusses, } \\
\text { Offenhalten von } \\
\text { autonomen } \\
\text { Artikulationschancen }\end{array}$ \\
\hline
\end{tabular}


Quelle: Eigene Zusammenstellung auf Basis von Ferree et al. (2002: 229); Gerhards et al. (1998: 37); Peters (2007: 188).

Die liberale Tradition normativer Öffentlichkeitsvorstellungen lässt sich mit der Spiegelmetapher auf den Punkt bringen. Die Funktion von Öffentlichkeit besteht demnach darin, das in der Gesellschaft vorhandene Spektrum an Positionen und Sprechern proportional zu ihrer jeweiligen Stärke naturgetreu abzubilden. Man kann innerhalb der liberalen Tradition eine eher elitäre Vorstellung und eine eher inklusive Vorstellung von Repräsentation unterscheiden (vgl. Ferree et al. 2002). Entscheidend ist in jedem Fall, dass die Repräsentation von Interessen und Positionen im Vordergrund steht und nicht besondere Anforderungen an die Qualität der Kommunikation oder ihr Ergebnis. Unter Wahrung eines Mindeststandards an gegenseitigem Respekt sind unterschiedliche, auch nichtdialogische Kommunikationsstile wie Verlautbarung und Agitation (vgl. Neidhardt 1994: 20) erlaubt. Debatten sollen abgeschlossen werden, sobald sich das Mehrheitsinteresse herauskristallisiert hat. Normative Öffentlichkeitsvorstellungen in der liberalen Tradition weisen zudem Ähnlichkeiten mit den betont nichtnormativen Konzepten von Öffentlichkeit innerhalb der soziologischen Systemtheorie auf, die auf die Ermöglichung gesellschaftlicher Selbstbeobachtung durch Öffentlichkeit abheben (vgl. etwa Luhmann 1996; Marcinkowski 1993). Auch wenn verschiedene systemtheoretische Konzeptionen das in Rede stehende System unterschiedlich benennen (Massenmedien, Publizistik, Öffentlichkeit oder Journalismus) und die systemische Primärfunktion dementsprechend auch unterschiedlich bestimmen, steht in jedem Fall die Vorstellung Pate, dass gesellschaftliche Kommunikationen nur durch die Systemlogik der Öffentlichkeit gefiltert der Gesellschaft zur Verfügung gestellt werden.

Die deliberative Tradition folgt demgegenüber eher der Metapher einer Diskussionsrunde. Möglichst unterschiedliche Akteure sollen an der öffentlichen Kommunikation beteiligt werden. Vor allem aber sollen sie dialogisch und respektvoll aufeinander eingehen und dabei ihre eigenen Positionen nicht nur behaupten, sondern begründen und einen zivilen Kommunikationsstil praktizieren. Auf diese Art und Weise sollen gesellschaftliche Konflikte eingehegt und in lösungsorientierte Debatten verwandelt werden. Das normative Ziel öffentlicher Kommunikation besteht darin, dass sich eine argumentativ erhärtete Mehrheitsmeinung herausbildet, wenn kein Konsens möglich ist (vgl. Habermas 1992; Peters 2007; Wessler 2008a). Der Prozess öffentlicher Deliberation stellt eine Alternative zum Verhandeln einerseits und zur reinen erfolgsorientierten Rhetorik andererseits dar. 
Öffentlicher Deliberation werden dabei positive Effekte sowohl für die Qualität politischer Urteile und Entscheidungen (kognitiver Gewinn) als auch für den Zusammenhalt der Diskutierenden zugeschrieben (sozialintegrativer Gewinn).

Die agonistische Tradition schließlich dreht sich um den freien Selbstausdruck und die Selbstermächtigung insbesondere marginaler und unterdrückter Gruppen und Gemeinschaften (vgl. Mouffe 1999; Sanders 1997). Öffentlichkeit stellt sich metaphorisch als bunter Protestmarsch dar. Robuste Konflikte über unterschiedliche Erfahrungen, Werte und Identitäten werden nicht nur als unvermeidlich, sondern als erwünscht angesehen. Öffentliche Kommunikation soll denn auch nicht zu einem besonderen Ziel führen, sondern die autonomen Artikulationschancen subalterner Gruppen und damit die Existenz von Gegenöffentlichkeiten dauerhaft offen halten. Das deliberative Modell von Öffentlichkeit wird in dieser Tradition häufig dafür kritisiert, dass es unzivile Ausdrucksformen abwerte und damit marginale Gruppen und militante Formen des Protests aus der Öffentlichkeit ausschließe. Emanzipativer Selbstausdruck umfasse aber notwendigerweise nichtargumentative Formen wie narrative Selbstoffenbarung, Rhetorik oder auch öffentliche Wertschätzung und Anerkennung (vgl. Young 2000).

Alle drei Traditionen normativer Öffentlichkeitstheorie sind empirisch anschlussfähig. Das heißt, dass sich die jeweils formulierten normativen Kriterien als Messlatten nutzen lassen, mit deren Hilfe die empirische Beschaffenheit von Öffentlichkeit(en) ermittelt und bewertet werden kann. In den folgenden Abschnitten wird diese Art von normativ angeleiteter und folgenreicher empirischer Öffentlichkeitsforschung beleuchtet. Dabei wird Deutschland jeweils im internationalen Vergleich betrachtet, um etwaige Besonderheiten der deutschen Öffentlichkeit offenzulegen.

\section{2. Öffentlichkeitsrelevante Medienstrukturen}

Hallin und Mancini (2004) unterscheiden in den europäischen und nordamerikanischen Demokratien drei Modelle des Zusammenspiels von Medien und Politik: neben dem demokratisch-korporatistischen Modell, das sich in West- und Nordeuropa, also auch in Deutschland findet, ein polarisiert-pluralistisches Modell, das in Südeuropa vorherrscht, und ein liberales Modell in den angelsächsischen Ländern. Sie machen das jeweils herrschende Öffentlichkeitsmodell an den Ausprägungen fest, die die Länder auf vier verschiedenen Makro-Dimensionen aufweisen. Da ist zunächst die historische Entwicklung der Medienmärkte, für Deutschland insbesondere die vergleichsweise frühe und weite 
Verbreitung von Tageszeitungen und die relative Stabilität und Akzeptanz öffentlichrechtlicher Rundfunkangebote auch nach der Einführung kommerzieller Fernseh- und Radioanbieter Mitte der 1980er Jahre.

Die zweite Dimension - politischer Parallelismus - betrifft das Ausmaß, zu dem sich politische Konfliktlinien im Feld der Medienanbieter abbilden. Der politische Parallelismus des deutschen Mediensystems ist gemäßigt. Bei den nationalen Qualitätszeitungen existiert mit „tageszeitung“, „Frankfurter Rundschau“, „Süddeutsche Zeitung“, „Frankfurter Allgemeine Zeitung“ und „Die Welt“ - eine politische Links-Rechts-Differenzierung nach Anbietern. Ähnliches gilt in gewissem Ausmaß für die Nachrichtenmagazine „Der Spiegel“ und „Focus“. Bei den Boulevardzeitungen dominiert eindeutig die rechts von der Mitte angesiedelte „Bild“. In den öffentlichen-rechtlichen Rundfunkanstalten ist bei aller verfassungsrechtlich geforderten Staatsferne ein gewisser Einfluss der politischen Parteien in den Aufsichtsgremien und der Personalpolitik unverkennbar. Die Mehrzahl der Vertreter in den Rundfunkräten stellen jedoch zumeist die gesellschaftlich relevanten Gruppen aus Kultur, Sport, Wirtschaft, Gewerkschaften, Wohlfahrt, Religion und der weiteren Zivilgesellschaft. Damit folgt Deutschland dem „civic model“ der Rundfunkaufsicht, das gesellschaftliche Kontrolle mit einem gewissen politischen Einfluss verbindet, ohne eine direkte Regierungsabhängigkeit des öffentlich-rechtlichen Rundfunks zu tolerieren, wie sie etwa in Südeuropa anzutreffen ist. Dadurch hat sich eine milde Form des externen Pluralismus zwischen den verschiedenen Landesrundfunkanstalten herausgebildet. In den übrigen Medienbereichen, insbesondere bei der in Deutschland traditionell bedeutenden Regionalpresse und im kommerziellen Fernsehen und Radio lässt sich eine politische Differenzierung auf Anbieterebene kaum erkennen. Politische Blogs, die etwa in den USA eine starke politische Rechts-Links-Polarisierung aufweisen, sind in Deutschland noch vergleichsweise schwach ausgeprägt.

Die dritte Dimension bei Hallin und Mancini (2004) betrifft den Grad der journalistischen Professionalisierung, wobei die Autoren neutrale und ausgewogene Berichterstattung nicht als die einzige Form von Professionalität gelten lassen, sondern auch begrenzt advokatorische Formen als professionell ansehen, sofern sie mit subjektiver Gemeinwohlorientierung und einem unter Journalisten geteilten Verständnis von Qualität einhergehen (Hallin/Mancini 2004: 41). Gemessen an dieser Definition sowie weiteren strukturellen Merkmalen - Qualität der Journalistenausbildung, Existenz von Selbstkontrollorganen etc. - ist die journalistische Professionalität in Deutschland vergleichsweise hoch. 
Viertens schließlich prägt das Ausmaß an staatlicher Intervention in den Medienmarkt das Öffentlichkeitsmodell. Jenseits der notwendigen Grundsicherung von Presse- und Informationsfreiheit spielen hier Regelungen zur institutionellen Absicherung von Medienvielfalt eine große Rolle. In Deutschland existieren Anti-Konzentrationsregeln im Bereich des kommerziellen Rundfunks sowie für den Besitz unterschiedlicher Medientypen (Presse, Radio, Fernsehen) durch ein und dasselbe Unternehmen. Zwar wurden diese Regeln bis zu einem gewissen Grad an die reale Entwicklung des Medienbesitzes angepasst, aber sie verhindern inzwischen wirksam die Entstehung eines Medienmonopols, wie es etwa in Italien zu beobachten war.

Die Medienstrukturen in Deutschland sind in mehrerer Hinsicht gemäßigt, gemischt und relativ stabil. Das Rundfunksystem vereinigt in Deutschland wie in den meisten europäischen Ländern kommerzielle und öffentlich-rechtliche Anbieter, wodurch die Marktorientierung im Mediensystem insgesamt geringer ausfällt als in Ländern mit rein kommerziellem System. Deutschland weist im internationalen Vergleich zusammen mit Dänemark und Norwegen die höchste öffentliche Pro-Kopf-Finanzierung des Rundfunks auf (jährlich rund 100 Euro); die USA bilden unter den westlichen Demokratien hier das Schlusslicht mit rund drei Euro öffentlicher Rundfunkfinanzierung pro Kopf und Jahr (Benson/Powers 2011: 61). Der Einfluss partikularer Interessen aus Wirtschaft und Politik auf das Medienangebot und insbesondere auf die politische Berichterstattung ist durch journalistische Selbstkontrolle, gesetzliche Konzentrationskontrolle sowie gesellschaftliche Aufsicht und föderale

Differenzierung des öffentlich-rechtlichen Rundfunks zumindest gebremster als in Südeuropa. Extern plurale, d.h. politisch identifizierbare Medienanbieter finden sich Seite an Seite mit intern pluralen und apolitischen Anbietern, so dass es zu keiner durchgängigen politischen Polarisierung im Mediensystem kommt.

3. Öffentlichkeitsrelevante Medienleistungen

\subsection{Die Sprecher}

Im Hinblick auf die in den Qualitätszeitungen zu Wort kommenden Sprecher sind in Deutschland staatliche Akteure stärker vertreten als in angelsächsischen Ländern (Ferree et al. 2002: 90; Wessler 2008b: 232), wo umgekehrt zivilgesellschaftliche Gruppen und einfache Bürger häufiger in den Qualitätszeitungen vertreten sind. Eine gewisse etatistische Tradition scheint sich also nach wie vor durchzusetzen, so dass die deutschen Qualitätszeitungen den Partizipations- und Empowerment-Forderungen des deliberativen bzw. des agonistischen 
Modells etwas weniger nachkommen als ihre angelsächsischen Pendants. Das in den deutschen Fernsehnachrichten zu Wort kommende Sprecherensemble unterscheidet sich dagegen kaum von dem Sprecherensemble in US-amerikanischen (und russischen) Fernsehnachrichten (Rinke/Wessler 2011). Allerdings zeigen sich in der Prominenz staatlicher Sprecher in den deutschen Fernsehnachrichten starke Unterschiede zwischen einzelnen Sendern: Die öffentlich-rechtliche ARD gibt staatlichen Sprechern deutlich mehr Gewicht als die kommerziellen Sender „RTL“ und „n-tv“. Auch insgesamt ist der Anteil politischer Themen in den Nachrichten der ARD deutlich höher als bei der kommerziellen Konkurrenz.

Das Internet bietet potentiell deutlich größere Artikulationschancen für Sprecher aus der Peripherie des politischen Systems, vor allem auch für subalterne und marginale Akteure, weil die technischen und finanziellen Voraussetzungen für die Publikation eigener Inhalte vergleichsweise gering sind. Allerdings ist es im Internet auch deutlich schwieriger, mit den eigenen Äußerungen gesamtgesellschaftliche Resonanz zu erzeugen. So zeigen Gerhards/Schäfer (2010: 149) in ihrer vergleichenden Fallstudie der Berichterstattung über die Genomentschlüsselung in Qualitätszeitungen und auf prominenten Webseiten, dass es im Sprecherensemble keine dramatischen Unterschiede zwischen beiden Foren gab. Auch bei der Bewertung der Genomforschung und bei den von den Sprechern verwendeten Interpretationsrahmen (Frames) boten die Webseiten keine größere Vielfalt der Meinungen und Deutungen. Allerdings fanden sich zum Teil erhebliche Unterschiede zwischen den deutschen und den US-amerikanischen Webseiten, wobei die deutschen Seiten ein breiteres Sprecherspektrum und mehr Kritik am Genomprojekt zur Verfügung stellten (Gerhards/Schäfer 2010). Die durch Suchmaschinen zugänglichen Webseiten stellen also insgesamt kein partizipativeres Forum dar als Qualitätszeitungen, unter anderem weil die von Suchmaschinen verwendeten Algorithmen die Verlinkung einer Webseite mit und von anderen Webseiten durch eine hohe Position in der Ergebnisliste belohnen und eine solche Vernetzung für ressourcenschwächere und subalterne Akteure nur schwer zu erreichen ist.

Das Internet bietet solchen Akteuren gleichwohl verbesserte Möglichkeiten, durch Webseiten, Blogs oder Social Media wie „Facebook“ oder „Twitter“ kleinere autonome Teilöffentlichkeiten auszubilden, wie sie vom agonistischen Öffentlichkeitsmodell gefordert werden. Sobald es aber um gesamtgesellschaftliche Beachtung und weiter reichenden Einfluss geht, sind gerade ressourcenschwächere zivilgesellschaftliche Akteure darauf angewiesen, dass ihre Online-Äußerungen selbst wieder durch die Massenmedien aufgegriffen und 
verstärkt werden. Die Beobachtung der Blogosphäre hat sich seit Mitte der 2000er Jahre als neue journalistische Recherchetechnik eingebürgert, die Wahrscheinlichkeit des „bottom-up intermedia agenda setting“ steigt, bei dem marginale und alternative Medien Öffentlichkeit für neue Themen generieren, die dann im Mainstream aufgegriffen werden. Als Beispiel für dieses Muster kann die „Guttenberg-Affäre“ gesehen werden, die in einer Fachöffentlichkeit initiiert und sodann durch Medienberichterstattung zur Online-Mobilisierung exponentiell in ihrer Öffentlichkeitswirksamkeit gesteigert wurde. In der Folge musste der deutsche Bundesverteidigungsminister, dem Plagiarismus in seiner Doktorarbeit nachgewiesen wurde, im Frühjahr 2011 zurücktreten. Die allgemeinere Frage allerdings, inwiefern der Einfluss von Online-Öffentlichkeiten auf das politische System in Häufigkeit und Folgenschwere zunimmt, bleibt einstweilen offen. Gleiches gilt auch für die Frage danach, ob der Aufstieg von OnlineÖffentlichkeiten mit einem Bedeutungsverlust für klassische massenmediale Öffentlichkeiten einhergeht.

\subsection{Die Qualität des Medieninhalts}

Mit Blick auf das Wie der öffentlichen Kommunikation, insbesondere die deliberative Qualität von Medieninhalten, weist die existierende Literatur keine bemerkenswerten Niveauunterschiede zwischen deutschen Medien und Medien in anderen westlichen Ländern aus. Sie lenkt den Blick vielmehr auf Besonderheiten der journalistischen Kultur. Viel Aufmerksamkeit hat dabei die Frage erhalten, wie einseitig oder ausgewogen die Medienberichterstattung ist. Eine Form, gegenläufige Positionen transparent zu machen, besteht darin, beide Seiten eines Konflikts in ein und demselben Zeitungsartikel oder ein und derselben Fernsehnachricht zu Wort kommen zu lassen. Diese Form debattenartiger Darstellung eines Disputs findet sich eher in (Teil-)Systemen mit internem Pluralismus, in denen einzelne Medien versuchen, den Meinungspluralismus intern abzubilden. So zeigen Ferree et al. (2002: 240) am Beispiel der Abtreibungsdebatte, dass die binnenplural strukturierten US-Qualitätszeitungen einen größeren Anteil an nachrichtlichen Artikeln mit widerstreitenden Meinungen aufweisen, während in den politisch stärker identifizierbaren deutschen Qualitätszeitungen häufiger Nachrichten mit nur einer Meinungsrichtung auftauchen. Dies verweist auf einen Unterschied in der journalistischen Arbeitsweise, bedeutet jedoch nicht, dass die deutschen Zeitungen deshalb grundsätzlich weniger deliberativ wären. Denn Ferree et al. (2002) weisen auch nach, dass in Debattenartikeln sowohl deliberative als auch respektlose und unzivile „Interaktionen“ zwischen den Sprechern häufiger vorkommen als in Artikeln mit nur einer Meinung. 
Auch die vergleichende Analyse der Deliberativität von Fernsehnachrichten von Rinke und Wessler (2011) findet in deutschen Sendern etwas weniger Nachrichtenbeiträge mit gegenläufigen Positionen (das Äquivalent zu Debattenartikeln in der Presse) und etwas weniger Sprecheräußerungen, die sich explizit auf eine gegenläufige Äußerung beziehen als in US-amerikanischen Fernsehsendern. Umgekehrt weisen die deutschen Fernsehnachrichten einen größeren Anteil von Beiträgen auf, in denen Debatten im Vorfeld einer bestimmten politischen Entscheidung präsentiert werden. In den US-Sendern bezieht sich ein größerer Teil der politischen Nachrichten gar nicht auf eine politische Entscheidung oder präsentiert Diskussionen im Anschluss an bereits getroffene Entscheidungen. Debatten im Vorfeld sind von größerem Wert für die Öffentlichkeit, weil sie es ermöglichen, dass sich Bürger und Entscheidungsträger in ihren Urteilen und Entscheidungen an den vorgebrachten Argumenten orientieren können. Auch bei den Fernsehnachrichten ergibt sich also ein differenziertes Bild, in dem sich verschiedene Qualitätskriterien gegenseitig aufheben und die Analyse Unterschiede in der Journalismuskultur und der journalistischen Arbeitsweise sichtbar macht. Der Vergleich der deutschen und US-amerikanischen Fernsehnachrichten mit Nachrichten in Russland ergibt demgegenüber einen klaren Deliberativitäts- und Inklusivitätsvorsprung für die beiden etablierteren Demokratien (Rinke/Wessler 2001). Und sowohl in den USA als auch in Deutschland schneiden die öffentlich organisierten Sender bei fast allen Qualitätskriterien besser ab als ihre kommerziellen Konkurrenten.

Aalberg et al. (2010) ergänzen diese Studien mit ihrer Untersuchung von Medieninhalten in sechs westlichen Ländern in einer Langzeitperspektive. Sie zeigen im Zeit- und Ländervergleich, dass der Grad der Kommerzialisierung eines Mediensystems negativ assoziiert ist mit dem Ausmaß politischer Informationen, die es bereitstellt: Je marktorientierter Medien sind, desto weniger leisten sie an politischer Berichterstattung. Dabei weisen die ländervergleichenden Befunde darauf hin, dass eine starke Stellung des öffentlich-rechtlichen Rundfunks im gesamten Mediensystem - so wie es in Deutschland der Fall ist - nicht etwa, wie häufig behauptet wird, eine Verabschiedung des privaten Rundfunks aus der aktuellen Nachrichtenberichterstattung nach sich zieht. Im Gegenteil bieten in diesen Ländern auch private Sender durchschnittlich mehr politische Berichterstattung an. Der allgemeine Standard hinsichtlich der Versorgung mit Nachrichten durch das Fernsehen insgesamt wird durch öffentlichen Rundfunk also angehoben. Zugleich ist in solchen Ländern der Anteil der regelmäßig Nachrichten konsumierenden Bevölkerung größer als in Ländern mit stärker kommerzialisierten Mediensystemen. 
Neben Nachrichten in Printmedien und Fernsehen sind es vor allem politische Fernsehtalkshows, die die Bürger mit gesellschaftsweit relevanten Themen in Berührung bringen und insofern für die Konstitution von Öffentlichkeit wichtig sind. Auch wenn es zur Qualität von Talkshows bislang keine international vergleichende Forschung gibt, kann man sagen, dass Deutschland in diesem Bereich ein differenziertes Angebot aufweist, das von Politikerauftritten in Unterhaltungstalkshows über die Polit-Talks am Abend („Hart aber fair“, „Maybrit Illner“ etc.) bis zu politischen Expertengesprächen wie z.B. dem „Presseclub“ reicht. Schultz (2006) zeigt auf, worin die öffentlichkeitsrelevante Qualität von politischen Fernsehtalkshows bestehen kann: in einem mittleren Niveau der journalistischen Intervention und im Stellen von Rechtfertigungsfragen, die die beteiligten Politiker dazu anhalten, ihre Positionen nicht nur zu äußern, sondern auch zu begründen. Ein zu großes Maß an Konfrontativität zwischen den Beteiligten ist dagegen der Akzeptanz unterschiedlicher möglicher Standpunkte abträglich (vgl. Mutz 2007).

4. Öffentlichkeitsrelevante Medienwirkungen

\subsection{Politisches Wissen}

In einem Vergleich der Inhalte und Wirkungen von Nachrichten in der Schweiz und den USA finden Iyengar et al. (2009), dass mehr „harte“ Nachrichten zu einem insgesamt höheren Niveau politischen Wissens in der Gesamtgesellschaft sowie zu einem stärkeren Zusammenhang von Nachrichtennutzung mit individuellem politischen Wissen führen. Damit eng verbunden ist der Befund, dass ein Mediensystem mit öffentlich-rechtlichem Einfluss Ungleichheiten hinsichtlich des politischen Wissens in der Gesellschaft zu verringern im Stande ist. Ähnlich kommen Curran et al. (2009) in einem Vergleich verschiedener Mediensystemmodelle in vier Ländern zu dem Schluss, dass ein durch einen öffentlichen Auftrag gelenktes öffentlich-rechtliches System Nachrichten im Durchschnitt mehr Aufmerksamkeit schenkt und diese prominenter platziert, was die Auseinandersetzung mit politischen Fragen in der Bevölkerung fördert und letztlich zu geringeren Ungleichheiten zwischen sozioökonomisch privilegierten und benachteiligten Bevölkerungsgruppen hinsichtlich des politischen Wissens führt (siehe auch Curran et al. 2010). Eine großangelegte Studie auf Basis von Umfragedaten aus allen EU-Mitgliedsländern (Popescu/Tóka 2009) wiederum macht deutlich, dass sich Fernsehnachrichten allgemein - unter anderem durch beiläufige bzw. unbeabsichtigte Rezeption - positiv auf das politische Wissen weniger politisch interessierter Bürger auswirken. Ebenso finden die Autoren, dass dieser positive Lerneffekt für die Rezeption von öffentlichen Sendern stärker ausgeprägt ist als für die 
Nutzung privater Sender. Öffentlicher Rundfunk kann diese Stärke dabei besonders ausspielen, wenn er von Finanzierung durch Werbung unabhängig ist, einen klaren Informationsschwerpunkt aufweist und als General-Interest-Programm breite Teile der Bevölkerung als Zielgruppe definiert - Kriterien, die der deutsche öffentlich-rechtliche Rundfunk zum größeren Teil erfüllt. Etwas detailliertere Ergebnisse zu der Frage nach Medienwirkungen auf politisches Wissen präsentieren Holtz-Bacha und Norris (2001). Sie bestätigen das Bild eines allgemein positiven Zusammenhangs der Nutzung öffentlichrechtlicher Sender mit politischem Wissen. Jedoch zeigen sie in ihrer Studie über 15 europäische Länder für Deutschland ein Muster, demzufolge die Nutzung von Nachrichtenformaten per se wichtiger ist für das Niveau politischen Wissens als eine Nutzung von öffentlich-rechtlichen Sendern.

Vor dem Hintergrund des dualen Rundfunksystems in Deutschland und seiner genannten Folgen ist eine spezielle Entwicklung der letzten Jahre relevant für die Entwicklung von Öffentlichkeit in Deutschland: die Entwicklung hin zu einem so genannten „high choice media environment“ (Prior 2005), in dem eine Vielzahl an Kanälen für die Bürger verfügbar ist und die als Ergebnis eines „neuen Strukturwandels der Öffentlichkeit“ bezeichnet werden kann (Imhof 2006). Mit dieser Entwicklung steigt die Relevanz voneinander verstärkenden Spiralprozessen (so genannte „reinforcing spirals“, vgl. Slater 2007), in denen kognitive und soziale Voraussetzungen, mit denen Menschen sich Medienangeboten zuwenden, zunehmend die Folgen der Mediennutzung vorbestimmen. Dies kann zu so genannten „virtuous circles“ (Norris 2000) führen, in denen Medien entsprechend prädestinierte Nutzer „belohnen“, beispielsweise mit Wissenszugewinnen. Die großen Wahlmöglichkeiten können aber auch zu Abwärtsspiralen führen, in denen die sozioökonomische Position von Individuen verfestigt wird bzw. die soziale Mobilität in der Gesellschaft durch die Struktur der Medienangebote und deren Nutzung insgesamt verringert wird.

\subsection{Politisches Vertrauen, politische Toleranz und bürgerschaftliches Engagement}

Mit Blick auf das politische Vertrauen der Bürger gegenüber dem politischen System und den Politikern ist zu vermuten, dass die Wahrscheinlichkeit von „Zynismusspiralen“ (Cappella/Jamieson, 1997) ansteigt, in denen eine abwertende Politikberichterstattung, beispielsweise über die vermeintlich rein eigennützigen Handlungsmotive von Politikern, ein zynisches Bürgerpublikum hervorbringt. Dieser allgemein in den entwickelten westlichen Demokratien zu beobachtende Trend dürfte in Deutschland jedoch durch den öffentlich- 
rechtlichen Rundfunk gedämpft sein, der sich Prozessen der Boulevardisierung von Politikberichterstattung bis zu einem gewissen Grad entzieht.

Im Hinblick auf die politische Polarisierung und Toleranz gegenüber abweichenden Meinungen sei daran erinnert, dass das deutsche Mediensystem durch einen mäßigen politischen Parallelismus geprägt ist (vgl. Hallin/Mancini 2004). Auf der Grundlage international vergleichender Untersuchungen kann davon ausgegangen werden, dass das deutsche Mediensystem die deutsche Bevölkerung daher relativ stark mit widerstreitenden politischen Ansichten konfrontiert (vgl. Goldman/Mutz 2011). Eine solche „exposure to disagreement“ hat sich in verschiedenen Studien als zuträglich für die Wahrnehmung politischer Opposition als legitimem Bestandteil demokratischer Prozesse erwiesen (Mutz 2007) sowie als eine zentrale demokratische Funktion von Massenmedien allgemein (Mutz/Martin 2001). Jedoch nähren die steigenden Wahlmöglichkeiten im politischen Informationssystem Deutschlands Befürchtungen einer ebenso ansteigenden selektiven Zuwendung zu Medieninhalten, die den eigenen politischen Vorlieben entsprechen. Solchermaßen erleichterte Selektivität in der Nutzung politischer Medienangebote birgt dabei das Potenzial einer „Cyber-Balkanisierung“(Kobayashi/Ikeda 2009) sowie die Gefahr zunehmender „enclave deliberation“ - voneinander isolierter Diskussionen politisch Gleichgesinnter. Erneut ist in diesem Zusammenhang jedoch anzunehmen, dass solche Tendenzen in der deutschen Öffentlichkeit, im Vergleich zu rein marktförmig organisierten Mediensystemen, geringer ausgeprägt sind (vgl. Sunstein 2007). Weiterhin werden solche Tendenzen zur Fragmentierung der deutschen Öffentlichkeit dadurch abgemildert, dass

- die Strukturen von Online-Medienangeboten jene etablierter massenmedialer Angebote zum großen Teil widerspiegeln;

- Menschen im Allgemeinen zwar selektive Zuwendung zu politisch genehmen Medieninhalten betreiben mögen, weniger aber eine selektive Vermeidung dissonanter Online-Medieninhalte (siehe Garrett 2009);

- die relativ geringe Polarisierung der politischen Eliten und der politischen Kultur in Deutschland einerseits politische Selbstidentifikationen von Bürgern weniger eindeutig und dauerhaft macht und andererseits eine auf einfachen Signalen basierende Identifikation der politischen Ausrichtung von Medienangeboten erschwert.

Schließlich sind auch die Folgen von Öffentlichkeitsstrukturen für das Niveau des politischen und bürgerschaftlichen Engagements in der Gesellschaft wiederholt Gegenstand empirischer 
Forschung geworden. So zeigt eine jüngere vergleichende Studie von 74 Ländern, dass eine öffentlich-rechtliche Organisation des Rundfunksystems die Wahlbeteiligung im Durchschnitt steigert, weil in Rundfunksystemen, die einem öffentlichen Auftrag dienen, die Informationskosten der Bürger systematisch gesenkt werden (Baek 2009). Wie die hinsichtlich Datenbasis und -analyse vermutlich stärkste empirische Studie zum Zusammenhang von Internetnutzung und politischer Mobilisierung in Deutschland zeigt, wird durch eine Nutzung des Internets die politische Mobilisierung der deutschen Bevölkerung zwar moderat gesteigert; diese Wirkung ist aber ungleich über die Bevölkerung verteilt, wodurch soziale Ungleichheiten hinsichtlich des Niveaus der politischen Aktivierung durch die Veränderung der gesellschaftlichen Kommunikationsstrukturen mit Aufkommen des Internets eher verstärkt als abgebaut werden (Kroh/Neiss 2009). Auch in dieser Hinsicht kann also von einer „reinforcing spiral“ gesprochen werden. Eine wichtige Ausnahme von diesem Trend sind allerdings die Ungleichheiten zwischen verschiedenen Altersgruppen hinsichtlich ihrer durchschnittlichen politischen Aktivierung: Junge Deutsche nutzen das Internet (bislang) häufiger als alte und erfahren hierdurch einen größeren Zuwachs an politischem Interesse als ältere Bürger (vgl. Kroh/Neiss 2009: 12). Das politische Interesse gleicht sich dadurch intergenerationell also ein Stück weit an.

\section{Fazit}

Bei aller gebotenen Vorsicht lassen sich die hier zusammengetragenen Erkenntnisse wie folgt zusammenfassen: Im Vergleich zu anderen westlichen Demokratien ist die Versorgung mit und die Nutzung von politischen Informationen sowie entsprechend auch das Wissen der Bevölkerung über politische Zusammenhänge in Deutschland relativ gut ausgeprägt. Dies ist unter anderem eine Folge des nach wie vor starken öffentlich-rechtlichen Rundfunks, der zu den am besten finanzierten Rundfunksystemen der Welt gehört. Im Hinblick auf die Medienleistungen stellt sich die deutsche Öffentlichkeit als etwas weniger inklusiv dar als die Öffentlichkeit anderer Länder (ressourcenschwache Akteure haben etwas schlechtere Zugangschancen). Bei der deliberativen Qualität des Medienangebots scheint es dagegen keine gravierenden Niveauunterschiede zu anderen etablierten Demokratien zu geben. Die deutsche Medienöffentlichkeit zeichnet sich dabei durch eine journalistische Kultur aus, die milde Formen des politischen Parallelismus mit relativ starker journalistischer Professionalisierung verbindet und deshalb gemäßigt advokatorische Formen des Journalismus umfasst. 
In einem „high choice media environment“ und unter Bedingungen der dynamischen medientechnologischen Entwicklung müssen die öffentlichkeitsrelevanten Leistungen und Wirkungen der Medien in Zukunft stärker für bestimmte Medientypen und Segmente der Bevölkerung differenziert analysiert werden. Dass die deutsche Öffentlichkeit dabei weniger als etwa in den USA in voneinander abgeschottete Enklaven politisch Gleichgesinnter zu zerfallen droht, kann zwar vermutet werden, ist aber nicht langfristig gesichert. Ebenso scheint das deutsche Öffentlichkeitsmodell extremen Formen des politischen Zynismus und einem drastischen Absinken der politischen Mobilisierung keinen Vorschub zu leisten; auch diese Entwicklungen sind jedoch empirisch weiter genau zu beobachten. Allgemeine Trendaussagen zur Entwicklung der deutschen Öffentlichkeit, häufig in Form von Verfallsthesen vorgebracht, sind dabei allerdings kaum hilfreich. Dies gilt gerade auch dann, wenn das normative Grundanliegen der Öffentlichkeitsanalyse unverändert aktuell bleibt: Medien und Demokratie im Öffentlichkeitsbegriff zusammenzudenken.

\section{Literatur}

Aalberg, Tori/van Aelst, Peter/Curran, James (2010): Media systems and the political information environment: A cross-national comparison. The International Journal of Press/Politics, 15, 3, S. 255-271. doi:10.1177/1940161210367422

Baek, Mijeong (2009): A comparative analysis of political communication systems and voter turnout. American Journal of Political Science, 53, 2, S. 376-393. doi:10.1111/j.15405907.2009.00376.x

Benson, Rodney/Powers, Matthew (2011): Public media and political independence: Lessons for the future of journalism from around the world. Report for Freepress. New York.

Calhoun, Craig (Hrsg.) (1992): Habermas and the public sphere. Cambridge/MA, London. Cappella, Joseph N./Jamieson, Kathleen Hall (1997) : Spiral of cynicism: The press and the public good. New York.

Curran, James/Iyengar, Shanto/Brink Lund, Anker/Salovaara-Moring, Inka (2009): Media system, public knowledge and democracy. European Journal of Communication, 24, 1, S. 526. doi:10.1177/0267323108098943 
Curran, James/Salovaara-Moring, Inka/Coen, Sharon/Iyengar, Shanto (2010): Crime, foreigners and hard news: A cross-national comparison of reporting and public perception. Journalism, 11, 1, S. 3-19. doi:10.1177/1464884909350640

Ferree, Myra M./Gamson, William A./Gerhards, Jürgen/Rucht, Dieter (2002): Shaping abortion discourse. Democracy and the public sphere in Germany and the United States. Cambridge.

Garrett, R. Kelly (2009): Politically motivated reinforcement seeking: Reframing the selective exposure debate. Journal of Communication, 59, 4, S. 676-699.

Gerhards, Jürgen (1993): Neue Konfliktlinien in der Mobilisierung öffentlicher Meinung. Eine Fallstudie. Opladen.

Gerhards, Jürgen/Neidhardt, Friedhelm/Rucht, Dieter (1998): Zwischen Palaver und Diskurs. Strukturen öffentlicher Meinungsbildung am Beispiel der deutschen Diskussion zur Abtreibung. Opladen/Wiesbaden.

Gerhards, Jürgen/Schäfer, Mike S. (2010): Is the Internet a better public sphere? Comparing newspapers and internet in Germany and the US. New Media and Society 12, 1, S. 143-160.

Goldman, Seth K./Mutz, Diana C. (2011): The friendly media phenomenon: A cross-national analysis of cross-cutting exposure. Political Communication, 28, 1, S. 42-66. doi:10.1080/10584609.2010.544280

Habermas, Jürgen (1990) [1962]: Strukturwandel der Öffentlichkeit. Untersuchungen zu einer Kategorie der bürgerlichen Gesellschaft. Frankfurt/Main.

Habermas, Jürgen (1992): Faktizität und Geltung. Beiträge zur Diskurstheorie des Rechts und des demokratischen Rechtsstaats. Frankfurt/Main.

Habermas, Jürgen (2008): Hat die Demokratie noch eine epistemische Dimension? Empirische Forschung und normative Theorie. In: Habermas, Jürgen: Ach, Europa. Kleine politische Schriften XI. Frankfurt/Main.

Hallin, Daniel C./Mancini, Paolo (2004): Comparing media systems: Three models of media and politics. Cambridge. 
Holtz-Bacha, Christina/Norris, Pippa (2001): “To entertain, inform, and educate”: Still the role of public television. Political Communication, 18, 2, S. 123-140. doi:10.1080/105846001750322943

Imhof, Kurt (2006): Mediengesellschaft und Medialisierung. Medien \& Kommunikationswissenschaft, 54, 2, S. 191-215.

Iyengar, Shanto/Hahn, Kyu S./Bonfadelli, Heinz/Marr, Mirko (2009): “Dark areas of ignorance” revisited: Comparing international affairs knowledge in Switzerland and the United States. Communication Research, 36, 3, S. 341-358. doi:10.1177/0093650209333024

Kobayashi, Tetsuro/Ikeda, Ken’ichi (2009): Selective exposure in political web browsing: Empirical verification of "cyber-balkanization” in Japan and the USA. Information, Communication \& Society, 12, 6, S. 929-953. doi:10.1080/13691180802158490

Kroh, Martin/Neiss, Hannes (2009): Internet access and political engagement: Self-selection or causal effect? Präsentiert auf dem Annual Meeting of the American Political Science Association. Toronto, Kanada.

Luhmann, Niklas (1996): Die Realität der Massenmedien. Opladen.

Marcinkowski, Frank (1993): Publizistik als autopoietisches System. Politik und Massenmedien. Eine systemtheoretische Analyse. Opladen.

Mouffe, Chantal (1999): Deliberative democracy or agonistic pluralism? Social Research, 66, 3, S. 745-758.

Mutz, Diana C. (2007): Effects of “in-your-face” television discourse on perceptions of a legitimate opposition. American Political Science Review, 101, 4, S. 621-635. doi:10.1017/S000305540707044X

Mutz, Diana C./Martin, Paul S. (2001): Facilitating communication across lines of political difference: The role of mass media. American Political Science Review, 95, 1, S. 97-114.

Neidhardt, Friedhelm (1994): Öffentlichkeit, öffentliche Meinung, soziale Bewegungen. In: Neidhardt, Friedhelm (Hrsg.): Öffentlichkeit, öffentliche Meinung, soziale Bewegungen. Kölner Zeitschrift für Soziologie und Sozialpsychologie. Sonderheft 34, S. 7-41. Opladen. Norris, Pippa (2000): A virtuous circle: Political communications in post-industrial democracies. New York. 
Peters, Bernhard (2007): Der Sinn von Öffentlichkeit. Herausgegeben von Hartmut Wessler, mit einem Vorwort von Jürgen Habermas. Frankfurt/Main.

Popescu, Marina/Tóka, Gabor (2009): Public television, private television and citizens’ political knowledge. Präsentiert auf der Konferenz "Party Competition: New Insights and Approaches,” European University Institute, Florenz, Italien.

Prior, Markus (2005): News vs. entertainment: How increasing media choice widens gaps in political knowledge and turnout. American Journal of Political Science, 49, S. 577-592. doi:10.1111/j.1540-5907.2005.00143.x

Rinke, Eike M./Wessler, Hartmut (2011): Comparing the deliberativeness of television news in Germany, the U.S., and Russia. Paper presented at the annual conference of the International Communication Association, Boston, May 2011.

Sanders, Lynn M. (1997): Against deliberation. Political Theory, 25, 3, S. 347-376.

Schultz, Tanjev (2006): Geschwätz oder Diskurs? Die Rationalität politischer Talkshows im Fernsehen. Köln.

Slater, Michael D. (2007): Reinforcing spirals: The mutual influence of media selectivity and media effects and their impact on individual behavior and social identity. Communication Theory, 17, S. 281-303. doi: 10.1111/j.1468-2885.2007.00296.x

Sunstein, Cass R. (2007): Republic.com 2.0. Princeton, NJ.

Wessler, Hartmut (2008a): Investigating deliberativeness comparatively. Political Communication 25, 1, S. 1-22.

Wessler, Hartmut (2008b): Mediale Diskursöffentlichkeiten im internationalen Vergleich ein Forschungsprogramm. In: Melischek, Gabriele/Seethaler, Josef/Wilke, Jürgen (Hrsg.): Medien \& Kommunikationsforschung im Vergleich. Grundlagen, Gegenstandsbereiche, Verfahrensweisen (S. 219-236). Wiesbaden.

Young, Iris M. (2000): Inclusion and democracy. New York. 
Stichwörter:

Agonismus

Deliberation

Liberale Tradition

Mediensystem

Politische Medienwirkungen

Politische Kommunikation

Sprecher 